\title{
1 Simulating Substrate Recognition and Oxidation in Laccases: From Description to Design
}

\author{
3 Maria Fátima Lucas, ${ }^{*}, \dagger, t, \nabla \odot$ Emanuele Monza, ${ }^{\dagger, \nabla}{ }^{\circ}$ Lise J. Jørgensen, ${ }^{\S}{ }^{\S}$ Heidi A. Ernst, ${ }^{\S}{ }^{\S}$ Klaus Piontek, \\ ${ }_{4}$ Morten J. Bjerrum, ${ }^{\$ \odot}$ Ángel T. Martinez, ${ }^{\perp \odot}$ Susana Camarero, ${ }^{\perp \odot}$ and Víctor Guallar, ${ }^{*},, \# \odot$ \\ $5{ }^{\dagger}$ Joint BSC-CRG-IRB Research Program in Computational Biology, Barcelona Supercomputing Center, Jordi Girona 29, E-08034 \\ 6 Barcelona, Spain \\ $7{ }^{\ddagger}$ Anaxomics Biotech, Balmes 89, E-08008 Barcelona, Spain \\ $8{ }^{\S}$ Department of Chemistry, University of Copenhagen, Universitetsparken 5, DK-2100 Copenhagen $\varnothing$, C., Denmark \\ 9 "University of Freiburg, Albertstraße 21, D-79104 Freiburg im Breisgau, Germany \\ ${ }_{10}{ }^{\perp}$ Centro de Investigaciones Biológicas, CSIC, Ramiro de Maeztu 9, E-28040 Madrid, Spain \\ 11 \#ICREA, Passeig Lluís Companys 23, E-08010 Barcelona, Spain \\ 12 S Supporting Information
}

13 ABSTRACT: To meet the very specific requirements 14 demanded by industry, proteins must be appropriately tailored. 15 Engineering laccases, to improve the oxidation of small 16 molecules, with applications in multiple fields, is, however, a 17 difficult task. Most efforts have concentrated on increasing the 18 redox potential of the enzyme, but in recent work, we have 19 pursued an alternate strategy to engineering these biocatalysts. 20 In particular, we have found that redesigning substrate binding 21 at the T1 pocket, guided by in silico methodologies, to be a 22 more consistent option. In this work, we evaluate the 23 robustness of our computational approach to estimate activity, 24 emphasizing the importance of the binding event in laccase r 25

\section{INTRODUCTION}

27 Laccases (EC 1.10.3.2) are multicopper oxidases whose 28 catalytic core is organized in two copper centers: the T1 site, 29 where substrates are oxidized, and the trinuclear cluster, where 30 molecular oxygen is reduced to water. The broad specificity of 31 these proteins, the use of oxygen as final electron acceptor, and 32 its conversion into water make them ideal candidates for 33 sustainable industrial processes. ${ }^{1}$ On the other hand, the T1 34 copper's redox potential $\left(\mathrm{E}_{\mathrm{T} 1}{ }^{\circ}<0.8 \mathrm{~V}\right)$ constitutes an upper 35 limit to their application. Although mediators are successfully 36 used to extend their activity toward high redox potential 37 substrates, ${ }^{2}$ trying to broaden the chemical space of these 38 proteins by increasing their redox potential is a challenging 39 strategy. ${ }^{3}$ The observation of a linear dependence between the 40 logarithm of the specificity constant $\left(k_{\text {cat }} / K_{\mathrm{M}}\right)$ and the one41 electron redox potential difference between laccase's T1 copper 42 site and phenolic substrates has for a long time fueled this 43 approach. ${ }^{4}$ However, exceptions can be found in the literature, 44 such as the oxidation of syringaldazine by Myceliophthora 45 thermophila $\left(\mathrm{MtL},{E^{\circ}}_{\mathrm{T} 1}^{\circ}=0.46 \mathrm{~V}\right)$, Rhizoctonia solani $\left(E_{\mathrm{T} 1}^{\circ}=\right.$ $460.73 \mathrm{~V})$, and Pycnoporus cinnabarinus $\left(\mathrm{PcL},{E^{\circ} \mathrm{T} 1}^{\circ} 0.79 \mathrm{~V}\right)$, 47 which was shown to be more favorable in the enzyme with the 48 lowest redox potential. ${ }^{5,6}$ In fact, in addition to the redox 49 potential difference, the oxidation rate is also controlled by the
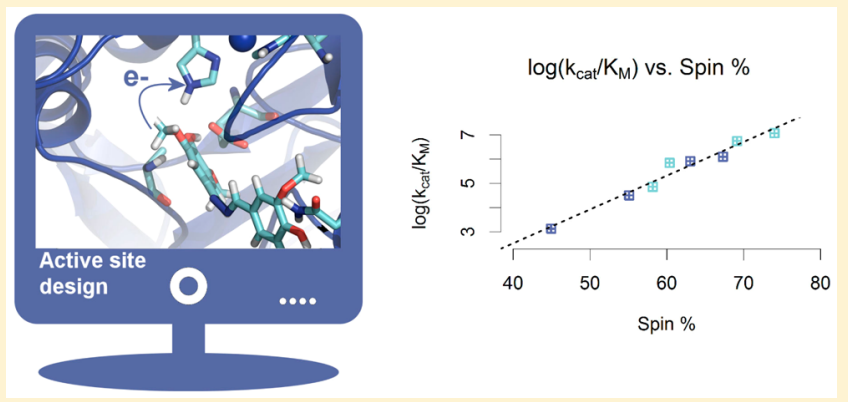

activity. Strengths and weaknesses of the protocol are discussed sequences and thus its significance in protein engineering.

binding event, ${ }^{7}$ which directly influences the electron transfer 50 (ET) driving force (shifting the energy levels of the substrate), 51 the electron coupling (determining the donor-acceptor 52 distance and their relative orientation), and the reorganization 53 energy (modulating substrate's solvent accessible area and 54 active site preorganization). To advance in this matter, we have 55 used a recently developed computational protocol ${ }^{7}$ that 56 combines the protein energy landscape exploration (PELE) 57 software $^{8,9}$ to map the laccase-substrate recognition process at 58 the $\mathrm{T} 1$ copper $(\mathrm{Cu})$ site, with quantum mechanics/molecular 59 mechanics (QM/MM) techniques, to score enzyme reactivity. 60

In this work, we systematically apply Monza et al.'s 61 methodology ${ }^{7}$ to study the oxidation of five substrates: 62 syringaldazine (SGZ; see Scheme 1) and four phenols with $63 \mathrm{~s} 1$ general formula $4 \mathrm{X}$-phenol $(\mathrm{X}=\mathrm{OH}, \mathrm{OMe}, \mathrm{Me}$, and $\mathrm{Cl}$; named 64 from this point on as $4 \mathrm{OH}, 4 \mathrm{MeO}, 4 \mathrm{Me}$, and $4 \mathrm{Cl}$, respectively) 65 by two fungal laccases: a high redox potential laccase from the 66 basidiomycete $\operatorname{PcL}\left(E_{\mathrm{T} 1}^{\circ}=0.79 \mathrm{~V}\right)$ and a medium redox 67 potential laccase from the ascomycete $\mathrm{MtL}\left(E_{\mathrm{T} 1}^{\circ}=0.46 \mathrm{~V}\right) . \quad 68$

The article is divided into two main sections: First, we 69 investigate the unusual reactivity profile of syringaldazine by 70

Received: November 27, 2016

Published: February 10, 2017 
Scheme 1. Chemical Structure of All Studied Substrates

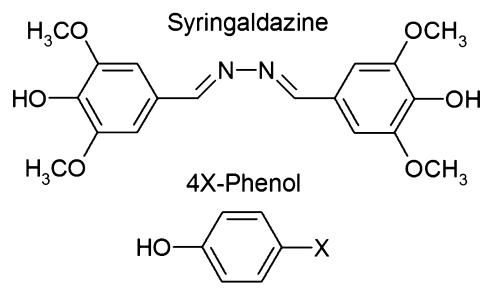

$\mathrm{X}=\mathrm{Cl}, \mathrm{Me}, \mathrm{MeO}, \mathrm{OH}$

$71 \mathrm{MtL}$ and PcL. Results confirm earlier findings on the 72 importance of the binding event in enzyme activity irrespective 73 of the enzyme's redox potential. ${ }^{7,10}$ Then, we perform a 74 systematic study of the oxidation of a series of related substrates 75 by MtL and PcL. These substrates were chosen because their 76 reactivity correlates with their driving force, ${ }^{4}$ approximated as 77 redox potential difference between electron acceptor and 78 donor, and to minimize potential changes in reactivity due to 79 substrate size and/or bulky substituents. The importance of a 80 correct description of the binding site (for example, the explicit 81 inclusion of crystal waters) and how clustering methods are 82 required to identify the most reactive positions are also 83 discussed.

\section{COMPUTATIONAL DETAILS}

85 Systems Setup. The crystal structures of PcL (2XYB.pdb) 86 and MtL (provided by Professor Bjerrum, to be published) 87 were prepared with Protein Preparation Wizard, ${ }^{11}$ PROPKA 88 utility, $^{12}$ and the $\mathrm{H}++$ Web server ${ }^{13}$ to determine the 89 protonation state of all titratable groups at $\mathrm{pH}$ 5. Five 90 substrates were prepared: SGZ and the four phenols derivatives $914 \mathrm{OH}, 4 \mathrm{MeO} 4 \mathrm{Me}$, and $4 \mathrm{Cl}$. The redox potential of these 92 phenols is $0.48 ; 0.66 ; 0.79 ; 0.90 \mathrm{~V}$, respectively. ${ }^{4}$ All substrates 93 were fully optimized with the density functional $\mathrm{M}^{2} 6^{14}$ with the $946-31 \mathrm{G}^{* *}$ basis set in an implicit solvent (modeled through the 95 Poisson-Boltzmann equation), and the atomic charges, 96 computed at the same level of theory by fitting the molecular 97 electrostatic potential, were used in the following force field 98 based PELE simulations.

99 Laccase-Substrate Recognition Process. To determine 100 the different binding modes of all substrates in the $\mathrm{T} 1 \mathrm{Cu}$ site of MtL and PcL, PELE simulations were performed. PELE, 101 which has been successfully applied to study enzymes, ${ }^{7,10,15-25} 102$ is a Monte Carlo algorithm that combines protein backbone 103 (elastic network model ${ }^{26}$ ) and ligand displacements (roto- 104 translations), followed by side chain repacking ${ }^{27}$ and all atom 105 minimization. The new positions are then accepted or rejected 106 through a Metropolis test based on energy differences 107 computed using an OPLS force field ${ }^{28}$ and a surface-generalized 108 Born implicit continuum solvent. ${ }^{29}$ In this study, the substrates 109 are manually placed close to the entrance of the $\mathrm{T} 1$ site and are 110 then free to move in a region $20 \AA$ within the $\mathrm{Cu}$ atom. An 111 example of such a trajectory can be seen in Supporting 112 Information. The results from local conformational searches for 113 SGZ in PcL and MtL (96 independent trajectories produced in 114 $48 \mathrm{~h}$ for each system), taken as an example, are visualized in 115 interaction plots (Figure 1). These contain all of the interaction $116 \mathrm{fl}$ energies of accepted minima against the distance of the center 117 of mass of the ligand to the $\mathrm{Cu}$ atom at the $\mathrm{T} 1$ site. Interaction 118 energies are computed as $E_{I N T}=E_{P S}-E_{P}-E_{S}$, where PS refers 119 to the protein $(\mathrm{P})$ substrate $(\mathrm{S})$ complex. 120

Structures of interest are then randomly selected up to $10 \AA 121$ of the $\mathrm{Cu}$ atom and within $5 \mathrm{kcal} / \mathrm{mol}$ of the lowest energy 122 value (red rectangle in Figure 1).

123

QM/MM Scoring. The selected structures obtained in the 124 previous PELE simulations were used to estimate the amount 125 of spin density transferred from the substrate to the $\mathrm{Cu}$ site. For 126 this, we have used a QM/MM scheme to model the whole 127 laccase-substrate complex which employs different levels of 128 theory to describe the system. QSite ${ }^{30}$ was used including in the 129 quantum region the entire $\mathrm{Cu}$ site (with equatorial and axial 130 ligands) as well as the substrate. The M06- $\mathrm{L}^{14}$ density 131 functional with the lacvp* basis set was used (LANL2DZ 132 effective core ${ }^{31}$ for the $\mathrm{Cu}$ atom and $6-31 \mathrm{G}^{*}$ for the rest of the 133 atoms). The remaining part of the protein was modeled with 134 molecular mechanics through an all-atom OPLS force field. A 135 five step geometry QM/MM optimization was carried out, and 136 Mulliken populations were computed to characterize the 137 fraction of spin density transferred from the substrate to the 138 $\mathrm{Cu}$ site. Previous experimental studies have shown that spin 139 densities correlate well with the ET driving force by establishing 140 if an unpaired electron is energetically more stable on the 141 donor or acceptor's molecular orbitals. ${ }^{32}$ This quick optimiza- 142

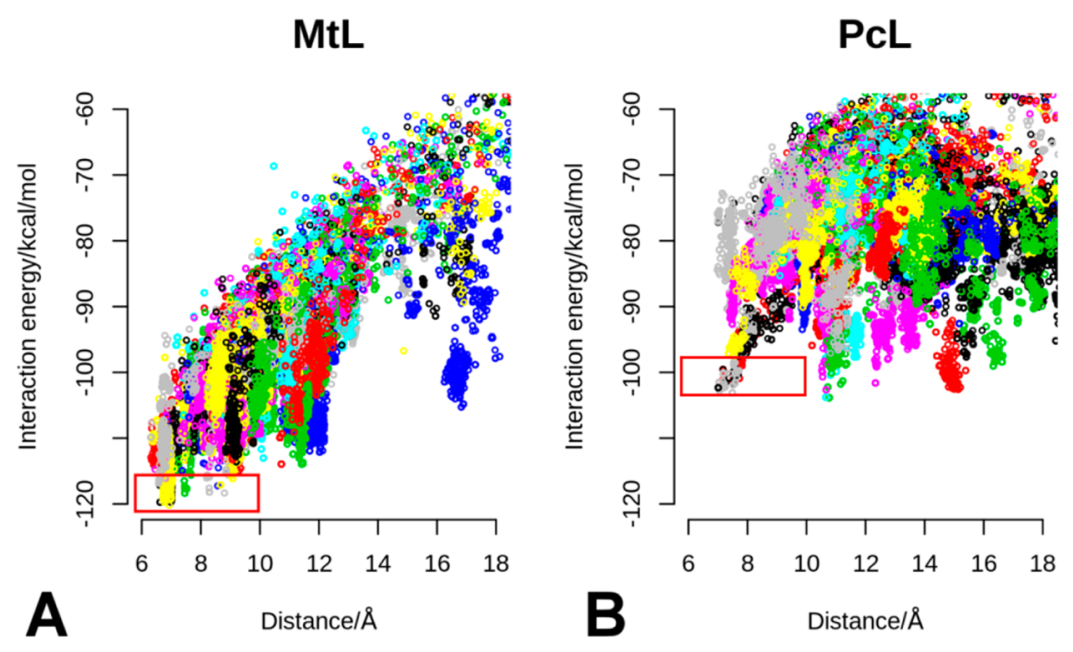

Figure 1. Interaction energies vs distance between the center of mass of SGZ and the T1 Cu atom in (A) MtL and (B) PcL. The different colors indicate independent single processor simulations. 


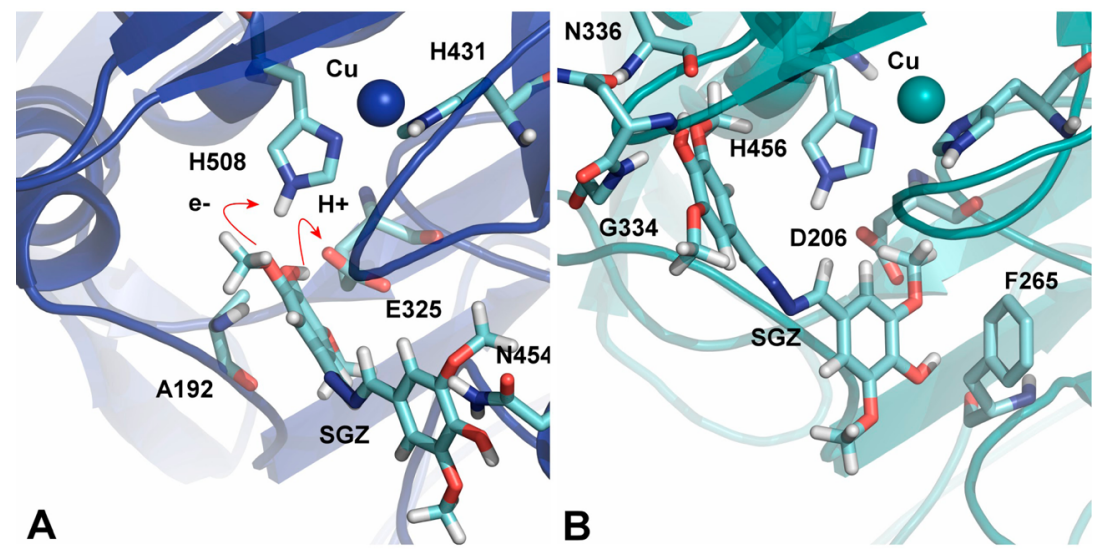

Figure 2. Representative binding modes for SGZ in (A) MtL and (B) PcL.

143 tion has been proven to be sufficient to obtain converged spin 144 densities. In laccases, moreover, the calculated spin population 145 has been shown to be largely invariant to changes in density 146 functionals, basis sets, and initial guesses. ${ }^{7}$

147 Molecular Dynamics. To ensure that two substrate 148 molecules can coexist in the active site, molecular dynamics 149 simulations were run for the PcL-4OH system, starting from 150 the configuration depicted in Figure S9. The ternary complex 151 was solvated with a $10 \AA$ buffer of water in an orthorhombic 152 box and neutralized, and $0.15 \mathrm{M} \mathrm{NaCl}$ was added. After 153 equilibration (default settings), $8 \times 5$ ns production runs at 154 constant temperature $(300 \mathrm{~K})$ and pressure $(1 \mathrm{~atm})$ were 155 performed with Desmond. ${ }^{33}$ The OPLS-2005 force-field ${ }^{28}$ and 156 the SPC explicit water model ${ }^{34}$ were used. The copper centers 157 were generated with the hetgrp_ffgen utility of Schrödinger, 158 using classical charges and crystal structure geometries. The 159 temperature was regulated with the Nosé-Hoover chain 160 thermostat $^{35}$ with a relaxation time of $1.0 \mathrm{ps}$, and the pressure 161 was controlled with the Martyna-Tobias-Klein barostat ${ }^{36}$ with 162 isotropic coupling and a relaxation time of $2.0 \mathrm{ps}$. The RESPA 163 integrator $^{37}$ was employed with bonded, near, and far time 164 steps of 2.0, 2.0, and 6.0 fs, respectively. A $9 \AA$ cutoff was used 165 for nonbonded interactions together with the smooth particle 166 mesh Ewald method. ${ }^{38}$

\section{RESULTS AND DISCUSSION}

168 Syringaldazine Oxidation by MtL and PcL. Although 169 unusual, there are some examples in the literature where lower 170 redox potential laccases display better $k_{\text {cat }}$ than their higher 171 redox potential counterparts. ${ }^{39}$ In particular, the oxidation of 172 syringaldazine by $\operatorname{MtL}\left(k_{\mathrm{cat}}=1100 \mathrm{~min}^{-1}\right)$ has been shown to 173 display improved kinetics over PcL's $\left(k_{\text {cat }}=180 \mathrm{~min}^{-1}\right){ }^{5}$ Here, 174 aiming at understanding these differences, we have investigated 175 the oxidation of SGZ by $\mathrm{MtL}$ and $\mathrm{PcL}$ using computational 176 tools. For this, 96 independent trajectories were produced, with 177 PELE, to establish the most favorable laccase-SGZ binding 178 modes. Then, 20 structures were randomly selected with an 179 energy-distance filter (see Computational Details) and QM/ $180 \mathrm{MM}$ calculations performed to assess the fraction of spin 181 density transferred from the substrate to the enzyme.

182 The interaction energy profiles for SGZ diffusion in MtL and 183 PcL are considerably different (Figure 1), and it is clear that 184 binding is more favorable (with a funnel-like profile and lower 185 interaction energies) in MtL than PcL. Additionally, minima in $186 \mathrm{MtL}$ are closer to the electron acceptor and better protected 187 from the solvent (in theory, lowering the ET coupling and reorganization energy, respectively) as seen in Figure S1. More 188 importantly, SGZ shows a better orientation for both electron 189 and proton transfer in MtL (Figure 2). 190 f 2

The differing binding of SGZ in MtL and PcL derives from 191 differences in the $\mathrm{T} 1$ pocket. MtL contains a large loop 192 involving residues 445 and 468 hosting Asn454 that anchors 193 the substrate in the position seen in Figure 2A. PcL, on the 194 contrary, has a much smaller loop of only 8 residues (as 195 opposed to the 23 in $\mathrm{MtL}$ ) involving residues 408 to 416.196 Another significant difference is the shorter loop hosting 197 residues 332 to 336 in $\mathrm{PcL}$ ( 364 to 371 in $\mathrm{MtL}$ ) that creates a 198 favorable environment to dock the polar hydroxyl group of 199 SGZ (Figure 2B). Finally, Ala192, which helps positioning the 200 substrate in the correct orientation in MtL's active site, is 201 located at the end of a loop that is preceded by an $\alpha$-helix 202 beginning in residue 179, which is not present in PcL (left side 203 of Figure 2A). In addition to the considerable differences seen 204 both in the interaction energy profiles, donor-acceptor 205 distances, solvent exposure, and binding orientation, QM/ 206 MM calculations also indicate higher spin transfer from SGZ to 207 MtL than in PcL (Figure 3).

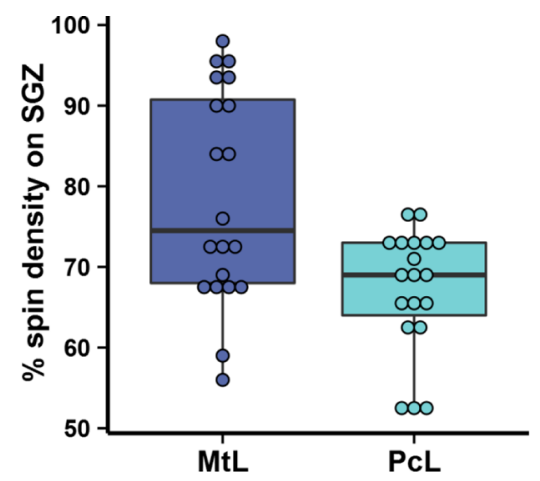

Figure 3. Distribution of SGZ spin densities in MtL and PcL in boxplot representations. In each distribution of data, we have minimum, first quartile, median, third quartile, and maximum. Each sphere is one computed spin density.

The computational results put in evidence the fact that 209 counterintuitive differences in SGZ's oxidation by these two 210 enzymes are highly related to the binding event. In MtL, SGZ 211 favors configurations hydrogen bonded (H-bonded) to the first 212 coordination His508 (facilitating ET) and simultaneous 213 interaction with Glu235 where proton transfer is expected to 214 
215 occur. $^{40}$ This will ensure optimal electronic coupling (shorter 216 donor-acceptor distance) and ET driving force (higher 217 substrate spin density, likely due to the proximity to the 218 catalytic Glu235). In PcL, however, SGZ interacts with the 219 backbone of residues Gly334 and Asn336 and with the side 220 chain of Phe 265 anchoring the substrate about $3 \AA$ away from 221 any of the T1 ligands. Indeed, Phe 265 has been described to 222 mark the boundary to the entrance channel to T1 site in the 223 high-redox potential laccase from Trametes versicolor, 1KYA, 224 with $66 \%$ sequence identity with $\mathrm{PcL} .{ }^{41}$ In this higher redox 225 enzyme, electron transfer must thus occur through a free space 226 jump (as none of the coordinated residues is close enough to 227 the substrate) or alternatively using other residues (which 228 necessarily imply a longer electron transfer path). These results 229 advocate for improved kinetics in $\mathrm{MtL}$, which is in good 230 agreement with experimental observations.

231 4X-Phenol Oxidation by MtL and PcL. Preliminary PELE 232 simulations for the 4X-phenols with PcL showed different 233 binding modes for each laccase-substrate system. However, $234 \mathrm{QM} / \mathrm{MM}$ calculations displayed poor correlation between the 235 amount of spin density computed on each substrate (20 236 structures selected) and the substrate's redox potential (Figure 237 S2 in Supporting Information). Further inspection of the most 238 favorable minima evidenced an important cluster of structures, 239 for all complexes, that included interaction with His456 (first 240 coordination sphere of the $\mathrm{Cu} \mathrm{T} 1$ site) but not with the 241 catalytic aspartic acid, which is expected to be the proton 242 acceptor (Figure S3A).

243 These structures exposed a region in PcL's binding pocket 244 with a volume comparable to that occupied by a water 245 molecule. Further inspection of the available laccases' crystal 246 structures that contained a phenolic substrate showed, for 247 example, that in Melanocarpus albomyces laccase in addition to 248 2,6-dimethoxyphenol it has a crystal water molecule precisely in 249 the position identified in the simulations (Figure S3B). For this 250 reason, we have repeated all calculations (PELE + QM/MM), 251 involving the phenols, including an extra water molecule in this 252 position. For each laccase-substrate pair, 240 independent $48 \mathrm{~h}$ 253 trajectories were produced (interaction energy profiles in 254 Figure S5), and 50 complex structures were randomly selected 255 for $\mathrm{QM} / \mathrm{MM}$ scoring. Computed spin densities for all systems 256 (depicted in Figures S6 and S7) offer a qualitative picture 257 where we can see the overall improved oxidation of compound $2584 \mathrm{OH}$ over $4 \mathrm{Cl}$ and that complexes with $\mathrm{PcL}$ display, on average, 259 higher spin transfer than with MtL. Next, we identified the site 260 with maximum substrate spin density, for each compound, by 261 clustering techniques. For this, computed spin densities (50 for 262 each system) were grouped using k-means and k-medoids, and 263 the obtained clusters (details can be found in SI Table S8 and 264 Figures S9 and S10) were then visually inspected. For both 265 laccases, the highest spin density cluster is also the one with 266 optimal catalytic contacts. The substrate is $\mathrm{H}$-bonded to the 267 catalytic histidine (first electron acceptor, coordinated to the $268 \mathrm{~T} 1$ copper) and to the water molecule present in the active site. 269 This is in turn hydrogen bonded to the catalytic Asp, the final 270 proton acceptor (Figure S4).

271 It follows that such a binding mode, besides being from an 272 excellent driving force (most likely due to the proximity of the 273 negatively charged catalytic base), provides both optimal 274 electron tunneling and proton abstraction. If only this cluster 275 of best oxidation position is taken into account, for each 276 system, a good correlation (above 95\%) between the computed spin density and the logarithm of the experimental specificity $277 \mathrm{f4}$ constants is obtained (Figure 4).

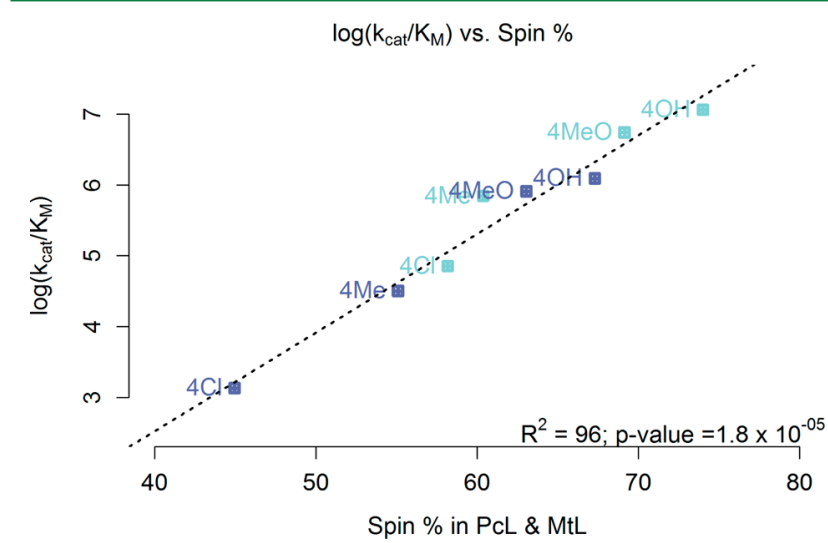

Figure 4. Computed average spin densities in the sites identified as the best oxidation positions in the T1 copper site for MtL and PcL vs experimental specificity constants ${ }^{4}$ for MtL (blue) and TvL (cyan).

Since specificity constants are not available for PcL, we 279 compared with another high redox potential enzyme from 280 Trametes villosa $\left(E_{\mathrm{T} 1}^{\circ}=0.78 \mathrm{~V}\right)$ which shares $70 \%$ identity 281 (homology modeling shows an identical ligand binding site to 282 PcL with only Phe164 replaced by an Ile in the binding pocket 283 entrance). These small differences may be responsible for the 284 lower correlation found for this system. To support the 285 hypothesis that, at high substrate concentration, the average 286 spin density at the best oxidizing site is correlated with 287 experimental specificity constants, we must ensure that 288 occupation of the best site is possible when other substrate 289 molecules are simultaneously bound at neighbor positions. For 290 this, we have performed eight independent $5 \mathrm{~ns}$ molecular 291 dynamics simulations and show that two ligands can, in fact, 292 concomitantly exist for at least 2 ns around their initial 293 positions (Figures S11 and S12). Furthermore, QM/MM 294 calculations confirm that the best site is the one oxidized by the 295 enzyme independently of the second substrate molecule. This 296 hypothesis is expected to be valid in systems where multiple 297 positions can be simultaneously occupied and where the 298 presence of a second ligand does not affect the oxidation of the 299 first (for example, charged substrates that change the 300 electrostatic environment of the substrate can affect the ET). 301

In any case, results confirm the potential of the in silico 302 protocol to discriminate laccase reactivity. Qualitatively scoring 303 enzyme activity is shown to be straightforward and the method 304 is robust enough to capture even unusual trends as with the 305 oxidation of SGZ by PcL and MtL. The present study further 306 validates a general methodology already used to explain 307 directed evolution experiments ${ }^{7,10}$ and computer-aided rational 308 design of laccases. ${ }^{15}$ In the latter, the oxidation of a poorly 309 reactive molecule was improved by introducing a negatively 310 charged residue to increase its oxidability. This was achieved by 311 tuning the binding event (PELE) and enhancing the spin 312 density (QM/MM). In the case of determining the oxidation 313 activity toward different substrates, it is clear that the problem 314 becomes much more complex. A correct description of the 315 binding site, namely, with the inclusion of essential water 316 molecules, is fundamental. Furthermore, we find that when 317 multiple binding modes exist, if they are noncompetitive, then 318 
319 the oxidation is mainly driven by the best oxidation position, 320 and clustering methods can help finding it.

321 An identical approach (PELE + QM/MM) was also recently 322 used to design a stable manganese peroxidase, ${ }^{20}$ which indicates 323 that the methodology benchmarked here can be easily extended 324 to other oxidoreductases. Nevertheless and as stated previously, 325 our aim is to develop cost-effective and reliable computational 326 strategies for protein engineering. In this context, we wish to 327 move away from rational design (where a few variants are 328 designed and validated experimentally) and instead provide an 329 exhaustive protein engineering protocol with reduced human 330 intervention. The goal is to either compute a large set of point 331 mutations and test the most promising experiments or identify 332 amino acids or regions of the protein where directed evolution 333 experiments should focus their effort. In this direction, we have 334 recently shown how the protocol benchmarked here can be 335 used for systematic sequence space search. In the work by 336 Giacobelli et al., ${ }^{42}$ we have used PELE to explore the binding in 337 the parental type. After identifying the most reactive laccase338 substrate conformation, over 400 mutants were evaluated with 339 a quick (less than $1 \mathrm{~h}$ in single processor per mutant) PELE 340 protocol. These calculations can be easily done in less than 1 341 day with 100 CPUs, providing an inexpensive alternative to 342 laboratory directed evolution. In fact, we have shown that while $343 \mathrm{QM} / \mathrm{MM}$ scoring (which is the most time-consuming step) 344 provides a reliable assessment it is possible to qualitatively 345 measure catalytic improvement, using only structural and force 346 field based parameters such as donor-acceptor distance, 347 catalytic contacts, and interaction energy. Final QM/MM 348 scoring, which takes $2-4 \mathrm{~h}$ on 4 CPUs, can easily be limited to 349 rerank the best $10-100$ variants, thus making this protocol 350 appropriate for academic and industrial laboratories, particularly 351 in a first approach to engineering a protein.

352 In conclusion, we demonstrate the robustness of the 353 computational method here benchmarked to accurately predict 354 the oxidation ability of laccases toward small molecules. We 355 discussed its potential to quickly score the activity of a large 356 number of protein sequences toward the oxidation of specific 357 substrates and thus the importance of in silico methodologies in 358 protein engineering.

\section{ASSOCIATED CONTENT}

360 S Supporting Information

361 The Supporting Information is available free of charge on the 362 ACS Publications website at DOI: 10.1021/acs.jctc.6b01158.

363 PELE simulations for SGZ with MtL and PcL; spin 364 distribution for 4X-phenols preliminary tests without a 365 water molecule; PELE simulations for 4X-phenols with 366 MtL and PcL; binding pocket comparison between PcL 367 and $\mathrm{MaL}$; binding positions for 4Cl-phenol in PcL; 368 substrate spin density boxplots for $4 \mathrm{X}$-phenols with PcL 369 and MtL; cluster analyses; and an example of two $4 \mathrm{OH}$ 370 minima in PcL (PDF)

371 Example of a PELE simulation (MPEG)

\section{AUTHOR INFORMATION}

\section{Corresponding Authors}

374 *(M.F.L.) E-mail: fati.lucas@gmail.com.

375 *(V.G.) E-mail: victor.guallar@bsc.es.

376 ORCID ${ }^{\circledR}$

377 Maria Fátima Lucas: 0000-0001-8672-9940

378 Emanuele Monza: 0000-0002-3443-9447
Lise J. Jørgensen: 0000-0003-0279-8653

Heidi A. Ernst: 0000-0001-5458-2454

Morten J. Bjerrum: 0000-0002-8410-627X

Ángel T. Martinez: 0000-0002-1584-2863

Susana Camarero: 0000-0002-2812-895X

Víctor Guallar: 0000-0002-4580-1114

\section{Author Contributions}

${ }^{\nabla}$ M.F.L. and E.M. contributed equally to this work.

\section{Funding}

387

This study was supported by the OxiDesign (CTQ2013-48287- 388 R) and NOESIS (BIO2014-56388-R) Spanish projects and the 389 INDOX (KBBE-2013-7-613549) EU-project.

Notes

The authors declare no competing financial interest.

\section{ABBREVIATIONS}

392

$\mathrm{E}^{\mathrm{o}}$, redox potential; PcL, Pycnoporus cinnabarinus laccase; $\mathrm{MtL}, 394$ Myceliophthora thermophila laccase; SGZ, syringaldazine; 4Cl, 395 4Cl-phenol; 4Me, 4Me-phenol; 4MeO, 4MeO-phenol; 4OH, 396 4OH-phenol; PELE, protein energy landscape exploration; 397 $\mathrm{QM} / \mathrm{MM}$, quantum mechanics/molecular mechanics

398

\section{REFERENCES}

399

(1) Cañas, A. I.; Camarero, S. Laccases and Their Natural Mediators: 400 Biotechnological Tools for Sustainable Eco-Friendly Processes. 401 Biotechnol. Adv. 2010, 28 (6), 694-705.

(2) Riva, S. Laccases: Blue Enzymes for Green Chemistry. Trends 403 Biotechnol. 2006, 24 (5), 219-226.

(3) Morozova, O. V.; Shumakovich, G. P.; Gorbacheva, M. A.; 405 Shleev, S. V.; Yaropolov, A. I. Blue" Laccases. Biochemistry 2007, 72406 (10), 1136-1150

(4) Tadesse, M. A.; D’Annibale, A.; Galli, C.; Gentili, P.; Sergi, F. An 408 Assessment of the Relative Contributions of Redox and Steric Issues to 409 Laccase Specificity towards Putative Substrates. Org. Biomol. Chem. 410 2008, 6 (5), 868-878.

411

(5) Li, K.; Xu, F.; Eriksson, K. E. Comparison of Fungal Laccases and 412 Redox Mediators in Oxidation of a Nonphenolic Lignin Model 413 Compound. Appl. Environ. Microbiol. 1999, 65 (6), 2654-2660. 414

(6) Xu, F.; Berka, R. M.; Wahleithner, J. A.; Nelson, B. A.; Shuster, J. 415 R.; Brown, S. H.; Palmer, A. E.; Solomon, E. I. Site-Directed Mutations 416 in Fungal Laccase: Effect on Redox Potential, Activity and pH Profile. 417 Biochem. J. 1998, 334 (1), 63-70.

(7) Monza, E.; Lucas, M. F.; Camarero, S.; Alejaldre, L. C.; Martínez, 419 A. T.; Guallar, V. Insights into Laccase Engineering from Molecular 420 Simulations: Toward a Binding-Focused Strategy. J. Phys. Chem. Lett. 421 2015, 6 (8), 1447-1453.

(8) Borrelli, K. W.; Vitalis, A.; Alcantara, R.; Guallar, V. PELE: 423 Protein Energy Landscape Exploration. A Novel Monte Carlo Based 424 Technique. J. Chem. Theory Comput. 2005, 1 (6), 1304-1311. 425

(9) Cossins, B. P.; Hosseini, A.; Guallar, V. Exploration of Protein 426 Conformational Change with PELE and Meta-Dynamics. J. Chem. 427 Theory Comput. 2012, 8 (3), 959-965.

(10) Pardo, I.; Santiago, G.; Gentili, P.; Lucas, F.; Monza, E.; 429 Medrano, F. J.; Galli, C.; Martínez, A. T.; Guallar, V.; Camarero, S. Re- 430 Designing the Substrate Binding Pocket of Laccase for Enhanced 431 Oxidation of Sinapic Acid. Catal. Sci. Technol. 2016, 6 (11), 3900- 432 3910.

(11) Sastry, G. M.; Adzhigirey, M.; Day, T.; Annabhimoju, R.; 434 Sherman, W. Protein and Ligand Preparation: Parameters, Protocols, 435 and Influence on Virtual Screening Enrichments. J. Comput.-Aided Mol. 436 Des. 2013, 27 (3), 221-234.

(12) Olsson, M. H. M.; Søndergaard, C. R.; Rostkowski, M.; Jensen, 438 J. H. PROPKA3: Consistent Treatment of Internal and Surface 439 Residues in Empirical pKa Predictions. J. Chem. Theory Comput. 2011, 440 7 (2), 525-537. 
442 (13) Gordon, J. C.; Myers, J. B.; Folta, T.; Shoja, V.; Heath, L. S.; 443 Onufriev, A. H. A Server for Estimating pKas and Adding Missing 444 Hydrogens to Macromolecules. Nucleic Acids Res. 2005, 33, W368445 W371.

446 (14) Zhao, Y.; Truhlar, D. G. The M06 Suite of Density Functionals 447 for Main Group Thermochemistry, Thermochemical Kinetics, Non448 covalent Interactions, Excited States, and Transition Elements: Two 449 New Functionals and Systematic Testing of Four M06 Functionals and 45012 Other Functionals. Theor. Chem. Acc. 2008, 119 (5-6), 525-525. 451 (15) Santiago, G.; de Salas, F.; Lucas, M. F.; Monza, E.; Acebes, S.; 452 Martinez, Á. T.; Camarero, S.; Guallar, V. Computer-Aided Laccase 453 Engineering: Toward Biological Oxidation of Arylamines. ACS Catal. 454 2016, 6 (8), 5415-5423.

455 (16) Babot, E. D.; Del Río, J. C.; Cañellas, M.; Sancho, F.; Lucas, F.; 456 Guallar, V.; Kalum, L.; Lund, H.; Gröbe, G.; Scheibner, K.; Ullrich, R.; 457 Hofrichter, M.; Martínez, A. T.; Gutiérrez, A. Steroid Hydroxylation by 458 Basidiomycete Peroxygenases: A Combined Experimental and 459 Computational Study. Appl. Environ. Microbiol. 2015, 81 (12), $4604130-4142$.

461 (17) Lucas, F.; Babot, E. D.; Cañellas, M.; del Río, J. C.; Kalum, L.; 462 Ullrich, R.; Hofrichter, M.; Guallar, V.; Martínez, A. T.; Gutiérrez, A. 463 Molecular Determinants for Selective C 25 -Hydroxylation of Vitamins 464 D 2 and D 3 by Fungal Peroxygenases. Catal. Sci. Technol. 2016, 6 (1), $465288-295$.

466 (18) Linde, D.; Pogni, R.; Cañellas, M.; Lucas, F.; Guallar, V.; 467 Baratto, M. C.; Sinicropi, A.; Sáez-Jiménez, V.; Coscolín, C.; Romero, 468 A.; Medrano, F. J.; Ruiz-Dueñas, F. J.; Martínez, A. T. Catalytic Surface 469 Radical in Dye-Decolorizing Peroxidase: A Computational, Spectro470 scopic and Site-Directed Mutagenesis Study. Biochem. J. 2015, 466 (2), $471253-262$.

472 (19) Molina-Espeja, P.; Cañellas, M.; Plou, F. J.; Hofrichter, M.; 473 Lucas, F.; Guallar, V.; Alcalde, M. Synthesis of 1-Naphthol by a 474 Natural Peroxygenase Engineered by Directed Evolution. ChemBio475 Chem 2016, 17 (4), 341-349.

476 (20) Acebes, S.; Fernandez-Fueyo, E.; Monza, E.; Lucas, M. F.; 477 Almendral, D.; Ruiz-Dueñas, F. J.; Lund, H.; Martinez, A. T.; Guallar, 478 V. Rational Enzyme Engineering Through Biophysical and Bio479 chemical Modeling. ACS Catal. 2016, 6 (3), 1624-1629.

480 (21) Lucas, M. F.; Guallar, V. An Atomistic View on Human 481 Hemoglobin Carbon Monoxide Migration Processes. Biophys. J. 2012, 482102 (4), 887-896.

483 (22) Saez-Jimenez, V.; Acebes, S.; Garcia-Ruiz, E.; Romero, A.; 484 Guallar, V.; Alcalde, M.; Medrano, F. J.; Martinez, A. T.; Ruiz-Duenas, 485 F. J. Unveiling the Basis of Alkaline Stability of an Evolved Versatile 486 Peroxidase. Biochem. J. 2016, 473 (13), 1917-1928.

487 (23) Sáez-Jiménez, V.; Acebes, S.; Guallar, V.; Martínez, A. T.; Ruiz488 Dueñas, F. J. Improving the Oxidative Stability of a High Redox 489 Potential Fungal Peroxidase by Rational Design. PLoS One 2015, 10 490 (4), e0124750.

491 (24) Miki, Y.; Pogni, R.; Acebes, S.; Lucas, F.; Fernández-Fueyo, E.; 492 Baratto, M. C.; Fernández, M. I.; de los Ríos, V.; Ruiz-Dueñas, F. J.; 493 Sinicropi, A.; Basosi, R.; Hammel, K. E.; Guallar, V.; Martínez, A. T. 494 Formation of a Tyrosine Adduct Involved in Lignin Degradation by 495 Trametopsis Cervina Lignin Peroxidase: A Novel Peroxidase 496 Activation Mechanism. Biochem. J. 2013, 452 (3), 575-584.

497 (25) Fernández-Fueyo, E.; Acebes, S.; Ruiz-Dueñas, F. J.; Martínez, 498 M. J.; Romero, A.; Medrano, F. J.; Guallar, V.; Martínez, A. T. 499 Structural Implications of the C-Terminal Tail in the Catalytic and 500 Stability Properties of Manganese Peroxidases from Ligninolytic Fungi. 501 Acta Crystallogr., Sect. D: Biol. Crystallogr. 2014, 70 (12), 3253-3265. 502 (26) Atilgan, A. R.; Durell, S. R.; Jernigan, R. L.; Demirel, M. C.; 503 Keskin, O.; Bahar, I. Anisotropy of Fluctuation Dynamics of Proteins 504 with an Elastic Network Model. Biophys. J. 2001, 80 (1), 505-515.

505 (27) Jacobson, M. P.; Kaminski, G. A.; Friesner, R. A.; Rapp, C. S. 506 Force Field Validation Using Protein Side Chain Prediction. J. Phys. 507 Chem. B 2002, 106 (44), 11673-11680.

508 (28) Kaminski, G. A.; Friesner, R. A.; Tirado-Rives, J.; Jorgensen, W. 509 L. Evaluation and Reparametrization of the OPLS-AA Force Field for 510 Proteins via Comparison with Accurate Quantum Chemical
Calculations on Peptides †. J. Phys. Chem. B 2001, 105 (28), 6474- 511 6487.

(29) Bashford, D.; Case, D. A. ChemInform Abstract: Generalized 513 Born Models of Macromolecular Solvation Effects. ChemInform 2001, 514 32 (8), no-no.

515

(30) Murphy, R. B.; Philipp, D. M.; Friesner, R. A. A Mixed 516 Quantum Mechanics/molecular Mechanics (QM/MM) Method for 517 Large-Scale Modeling of Chemistry in Protein Environments. J. 518 Comput. Chem. 2000, 21 (16), 1442-1457.

(31) Hay, P. J.; Jeffrey Hay, P.; Wadt, W. R. Ab Initio Effective Core 520 Potentials for Molecular Calculations. Potentials for the Transition 521 Metal Atoms Sc to Hg. J. Chem. Phys. 1985, 82 (1), 270.

(32) Artz, K.; Williams, J. C.; Allen, J. P.; Lendzian, F.; Rautter, J.; 523 Lubitz, W. Relationship between the Oxidation Potential and Electron 524 Spin Density of the Primary Electron Donor in Reaction Centers from 525 Rhodobacter Sphaeroides. Proc. Natl. Acad. Sci. U. S. A. 1997, 94 (25), 526 $13582-13587$.

(33) Bowers, K.; Chow, E.; Xu, H.; Dror, R.; Eastwood, M.; 528 Gregersen, B.; Klepeis, J.; Kolossvary, I.; Moraes, M.; Sacerdoti, F.; 529 Salmon, J.; Shan, Y.; Shaw, D. Scalable Algorithms for Molecular 530 Dynamics Simulations on Commodity Clusters. In ACM/IEEE SC 531 2006 Conference (SC'06); Horner-Miller, B., Ed.; ACM: New York, 532 2006.

(34) Toukan, K.; Rahman, A. Molecular-Dynamics Study of Atomic 534 Motions in Water. Phys. Rev. B: Condens. Matter Mater. Phys. 1985, 31535 (5), 2643-2648.

(35) Nosé, S.; Shuichi, N. A Unified Formulation of the Constant 537 Temperature Molecular Dynamics Methods. J. Chem. Phys. 1984, 81538 (1), 511.

(36) Martyna, G. J.; Tobias, D. J.; Klein, M. L. Constant Pressure 540 Molecular Dynamics Algorithms. J. Chem. Phys. 1994, 101 (5), 4177. 541

(37) Tuckerman, M.; Berne, B. J.; Martyna, G. J. Reversible Multiple 542 Time Scale Molecular Dynamics. J. Chem. Phys. 1992, 97 (3), 1990. 543

(38) Essmann, U.; Perera, L.; Berkowitz, M. L.; Darden, T.; Lee, H.; 544 Pedersen, L. G. A Smooth Particle Mesh Ewald Method. J. Chem. Phys. 545 1995, 103 (19), 8577.

(39) Xu, F.; Palmer, A. E.; Yaver, D. S.; Berka, R. M.; Gambetta, G. 547 A.; Brown, S. H.; Solomon, E. I. Targeted Mutations in a Trametes 548 Villosa Laccase. Axial Perturbations of the T1 Copper. J. Biol. Chem. 549 1999, 274 (18), 12372-12375.

550

(40) Galli, C.; Madzak, C.; Vadalà, R.; Jolivalt, C.; Gentili, P. 551 Concerted Electron/proton Transfer Mechanism in the Oxidation of 552 Phenols by Laccase. ChemBioChem 2013, 14 (18), 2500-2505. 553

(41) Bertrand, T.; Jolivalt, C.; Briozzo, P.; Caminade, E.; Joly, N.; 554 Madzak, C.; Mougin, C. Crystal Structure of a Four-Copper Laccase 555 Complexed with an Arylamine: Insights into Substrate Recognition 556 and Correlation with Kinetics. Biochemistry 2002, 41 (23), 7325- 557 7333.

(42) Giacobelli, V.; Monza, E.; Lucas, M. F.; Pezzella, C.; Piscitelli, 559 A.; Guallar, V.; Sannia, G. Repurposing Designed Mutants: A Valuable 560 Strategy for Computer-Aided Laccases Engineering. The Case of 561 POXAlb. Catal. Sci. Technol. 2017, 7, 515. 